\title{
AVALIAÇÃO DOS FLUXOS DAS PRINCIPAIS ESPÉCIES QUIMICAS DISSOLVIDAS NO RIO SOROCABA (SP)
}

\author{
Alexandre Martins FERNANDES ${ }^{1}$, Fabiano Tomazini da CONCEIÇÃO ${ }^{2}$, Eder Paulo \\ SPATTI JUNIOR ${ }^{2}$, Edvaldo GUEDES JUNIOR ${ }^{2}$, Diego de Souza SARDINHA ${ }^{3}$, Jefferson \\ MORTATTI ${ }^{4}$
}

(1) Departamento de Engenharia Civil, Faculdade de Engenharia de Bauru, Universidade Estadual Paulista "Júlio de Mesquita Filho". Avenida Engenheiro Luiz Edmundo Carrijo Coube, 14-01. CEP 17033-360. Bauru, SP. Endereço eletrônico: alefernandes1966@yahoo.com.br.

(2) Instituto de Geociências e Ciências Exatas, Universidade Estadual Paulista "Júlio de Mesquita Filho". Avenida 24-A, 1515. CEP 13506-900. Caixa Postal 178. Rio Claro, SP. Endereços eletrônicos: ftomazini@ rc.unesp.br, ederspatti@ hotmail.com, jr.guedes@ hotmail.com.

(3) Instituto de Ciência e Tecnologia, Universidade Federal de Alfenas. BR 267, km 533, Rodovia José Aurélio Vilela, 11.999, Cidade Universitária. CEP: 37715-400. Poços de Caldas, MG. Endereço eletrônico: diegosardinha@ yahoo.com.br.

(4) Centro de Energia Nuclear na Agricultura, Universidade de São Paulo. Avenida Centenário, 303. CEP: 13416-970. Caixa Postal 96. Piracicaba, SP. Endereço eletrônico: jmortatt@cena.usp.br.

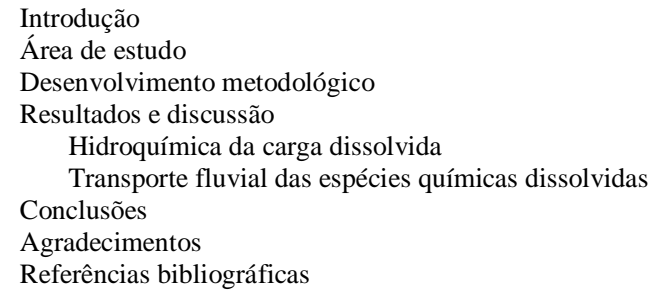

\begin{abstract}
RESUMO - O presente trabalho teve por objetivo realizar a caracterização hidroquímica das principais espécies químicas dissolvidas no Rio Sorocaba, bem como avaliar seus fluxos em um ciclo hidrológico e nos períodos seco e chuvoso. Entre junho 2009 e junho 2010 foram coletadas 12 amostras de águas fluviais, que apresentaram pH próximo à neutralidade (de 6,9 a 7,3) e uma variação sazonal marcante de condutividade elétrica, temperatura e concentração das espécies químicas estudadas. Os modelos de potência ajustados à relação concentração x vazão foram significativos $(p<0,01)$ e indicaram uma associação de $\mathrm{Ca}^{2+}, \mathrm{Mg}^{2+}, \mathrm{K}^{+}, \mathrm{HCO}_{3}{ }^{-}$e TDS a aportes difusos e de $\mathrm{Na}^{+}, \mathrm{NO}_{3}{ }^{-}$e $\mathrm{PO}_{4}{ }^{3-}$ a aportes pontuais, sem identificar claramente os aportes predominantes de $\mathrm{SiO}_{2}, \mathrm{Cl}^{-}$e $\mathrm{SO}_{4}{ }^{2-}$. Propôs-se a análise comparativa dos expoentes dos modelos ajustados, que corroborou as associações anteriores e relacionou $\mathrm{SiO}_{2}, \mathrm{Cl}^{-}$e $\mathrm{SO}_{4}{ }^{2-}$ a uma mistura de fontes. A contribuição da carga dissolvida do Rio Sorocaba para o Médio Tietê foi de 392,16x $10^{3} \mathrm{t} \mathrm{a}^{-1}$, sendo as mais significativas as de $\mathrm{HCO}_{3}{ }^{-}$e $\mathrm{SiO}_{2}$, seguidas de $\mathrm{Ca}^{2+}, \mathrm{Na}^{+}, \mathrm{Cl}^{-}, \mathrm{SO}_{4}{ }^{2-}, \mathrm{NO}_{3}{ }^{-}, \mathrm{K}^{+}, \mathrm{Mg}^{2+}$ e $\mathrm{PO}_{4}^{3-}$, ordem que se manteve nos períodos seco e chuvoso, com exceção do $\mathrm{NO}_{3}{ }^{-}$que apresentou carga semelhante nos três períodos.
\end{abstract}

Palavras-chave: bacia de drenagem, relação concentração x vazão, transporte fluvial,

ABSTRACT - FLUXES EVALUATION OF MAIN CHEMICAL SPECIES DISSOLVED IN THE SOROCABA RIVER (SP). This study aimed to characterize the hydrochemistry of the main chemical species dissolved in the Sorocaba River, as well as evaluate their fluxes in a hydrological cycle and during dry and rainy periods. Between June 2009 and June 2010, twelve river water samples were collected, which showed near neutral $\mathrm{pH}$ (6.9 to 7.3) and a marked seasonal variation of conductivity, temperature and concentration of chemical species studied. The regression models adjusted to concentration $\mathrm{x}$ discharge relationship were significant $(p<0,01)$ and indicated an association of $\mathrm{Ca}^{2+}, \mathrm{Mg}^{2+}, \mathrm{K}^{+}, \mathrm{HCO}_{3}{ }^{-}$and TDS to diffuse sources and $\mathrm{Na}^{+}, \mathrm{NO}_{3}{ }^{-}$and $\mathrm{PO}_{4}{ }^{3-}$ the punctual sources, without clearly identifying the predominant contributions of $\mathrm{SiO}_{2}, \mathrm{Cl}^{-}$and $\mathrm{SO}_{4}{ }^{2-}$. A comparative analysis of the exponents of the adjusted models proposed, which confirmed previous associations and related $\mathrm{SiO}_{2}, \mathrm{Cl}^{-}$and $\mathrm{SO}_{4}{ }^{2-}$ to a mix of sources. The dissolved load contribution of the Sorocaba River to Middle Tietê basin was $392.16 \times 10^{3} \mathrm{t} \mathrm{a}^{-1}$, the most significant being those of $\mathrm{HCO}_{3}{ }^{-}$and $\mathrm{SiO}_{2}$, followed by $\mathrm{Ca}^{2+}, \mathrm{Na}^{+}, \mathrm{Cl}^{-}, \mathrm{SO}_{4}{ }^{2-}, \mathrm{NO}_{3}{ }^{-}, \mathrm{K}^{+}, \mathrm{Mg}^{2+}$ and $\mathrm{PO}_{4}{ }^{3-}$, order maintained in the dry and rainy periods, with exception of $\mathrm{NO}_{3}{ }^{-}$ which showed similar charge in the three periods.

Keywords:

\section{INTRODUÇÃO}

Os cursos de água refletem a dinâmica da bacia de drenagem onde se inserem, compreendendo seus atributos naturais e a interrelação de seus vários componentes, como solo, água, cobertura vegetal, geologia; bem como as alterações antrópicas a que estão sujeitos (Jenkins et al., 1994). Ao longo da história humana, a água tem sido um recurso fundamental para o desenvolvimento das sociedades, e entre suas diversas utilizações, encontra-se como corpo receptor para diluir poluentes, o que tem afetado sobremaneira sua qualidade, particularmente em regiões onde a influência humana tem se intensificado nas últimas décadas (Carpenter et al., 1998). Em bacias hidrográficas densamente povoadas, 
industrializadas e/ou com agricultura intensa, a disponibilidade de água torna-se restrita, uma vez que sua qualidade é afetada pelo descarte de efluentes domésticos e industriais sem tratamento, bem como pelos insumos agrícolas lixiviados pelo escoamento superficial nos eventos de chuva, principalmente fertilizantes fosfatados e defensivos agrícolas.

O fluxo fluvial de elementos dissolvidos é alimentado pelos processos de intemperismo químico das rochas que compõem a bacia de drenagem, onde a dissolução ou hidrólise dos minerais primários das rochas liberam os elementos solúveis que são lixiviados pela água de drenagem (Probst, 1992), sendo que esses processos são fortemente influenciados pela natureza dos minerais primários, do clima, das influências da biosfera e da hidrodinâmica, entre outros (Martini \& Chesworth, 1992). Outras fontes de elementos/compostos para as águas superficiais são os aportes atmosféricos relacionados principalmente à precipitação e, em certas regiões, pela poluição antrópica (industrial, agrícola e/ou doméstica), que chegam ao rio de forma pontual ou difusa (Probst, 1992).

$\mathrm{O}$ interesse em investigar as cargas fluviais dissolvidas para encontrar a natureza e a composição da matéria transportada pelos rios inicia-se na década de 1960 (Barth, 1961; Garrels \& Mackenzie, 1967), intensificando-se nos anos 1970 (Gibbs, 1970, 1971), com estudos voltados para identificar a origem e os fatores de controle da carga dissolvida bem como avaliar e quantificar o transporte fluvial de íons majoritários dissolvidos, como, por exemplo, os trabalhos desenvolvidos por Martin \& Meybeck (1979), Stallard \& Edmond (1983, 1987), Kattan \& Probst (1986), Meybeck (1986), Probst et al. (1992a), Mortatti et al. (1994), Boeglin \& Probst (1996), Li \& Zhang (2008), Edet et al. (2013) e Laraque et al. (2013), dentre outros. No Estado de São Paulo, os estudos sobre a dinâmica da carga fluvial dissolvida são mais recentes, destacando-se os realizados nas bacias do Rio Corumbataí (Conceição \& Bonotto, 2002, 2003, 2004), Alto Sorocaba (Sardinha et al., 2010),
Tietê (Mortatti et al., 2008), Ribeirão do Meio (Conceição et al., 2010), Piracicaba (Mortatti et al., 2012), Jundiaí e Capivari (Oliveira et al., 2014).

A situação dos recursos hídricos no Estado de São Paulo mostra-se preocupante, onde a deterioração das fontes de água relacionadas ao crescimento e diversificação das atividades humanas e as consequentes alterações que ocorrem nas bacias de drenagem tem tornado cada vez mais difícil a identificação de corpos de água que atendam suas funções biológicas e, principalmente, que sirvam de mananciais para captação e abastecimento da população. As perspectivas para o futuro são ainda mais preocupantes, considerando modelo de desenvolvimento econômico vigente e os índices de crescimento populacional (Silva, 1994).

A bacia do Rio Sorocaba drena uma região cuja ocupação foi iniciada no século XVII e que passou por sucessivos ciclos de desenvolvimento e diversificação das atividades humanas. A partir da década de 1970, o desenvolvimento dessa região foi alavancado pela política de descentralização industrial da metrópole de São Paulo sem, no entanto, apresentar uma vocação específica. Atualmente, a bacia de drenagem apresenta áreas de pastagens degradadas e uma agricultura variada, com destaque para milho e cana-de-açúcar, e um parque industrial em expansão, principalmente nas cidades de Sorocaba e Votorantim, onde a presença da mineração e da indústria do cimento já se encontra consolidada (IPT, 2006).

O estudo da bacia do Rio Sorocaba ganha importância, pois além da pressão antrópica a que está sujeita, caracteriza-se como o principal manancial para captação e para abastecimento de água dos municípios presentes em sua área de drenagem, atendendo a mais de 1.000 .000 de habitantes (IPT, 2006). Nesse sentido, o presente trabalho tem por objetivo avaliar os fluxos das principais espécies químicas dissolvidas no Rio Sorocaba, junto à foz, no município de Laranjal Paulista - SP, a partir de sua caracterização hidroquímica em um ciclo hidrológico e nos períodos seco e chuvoso.

\section{ÁREA DE ESTUDO}

A bacia do Rio Sorocaba está localizada na região sudeste do Estado de São Paulo, Brasil, entre os paralelos $23^{\circ}$ e $24^{\circ}$ de latitude Sul e $47^{\circ}$ e $48^{\circ}$ de longitude Oeste, e apresenta uma área 
de drenagem de $5.269 \mathrm{~km}^{2}$, que abrange 18 municípios e uma população total de aproximadamente 1,2 milhões de habitantes (Figura 1). Esta bacia hidrográfica comporta um parque industrial diversificado composto por aproximadamente 1850 empreendimentos, onde se destacam os setores: têxtil, mecânico, metalúrgico, alimentício, curtumes e engenhos de aguardente. Considerado o afluente mais importante da margem esquerda da bacia do
Médio Tietê, percorre $227 \mathrm{~km}$ em direção noroeste até sua confluência com o Rio Tietê. A vegetação original caracteriza-se por matas, capoeiras, campos, cerrados e vegetação de várzea, da qual restam apenas $2 \%$. O atual uso da terra apresenta um predomínio de pastagens e campos antrópicos (77\%), seguido por culturas agrícolas (14\%), reflorestamento (3\%) e áreas urbanas (4\%) (IPT, 2006; IBGE, 2010).

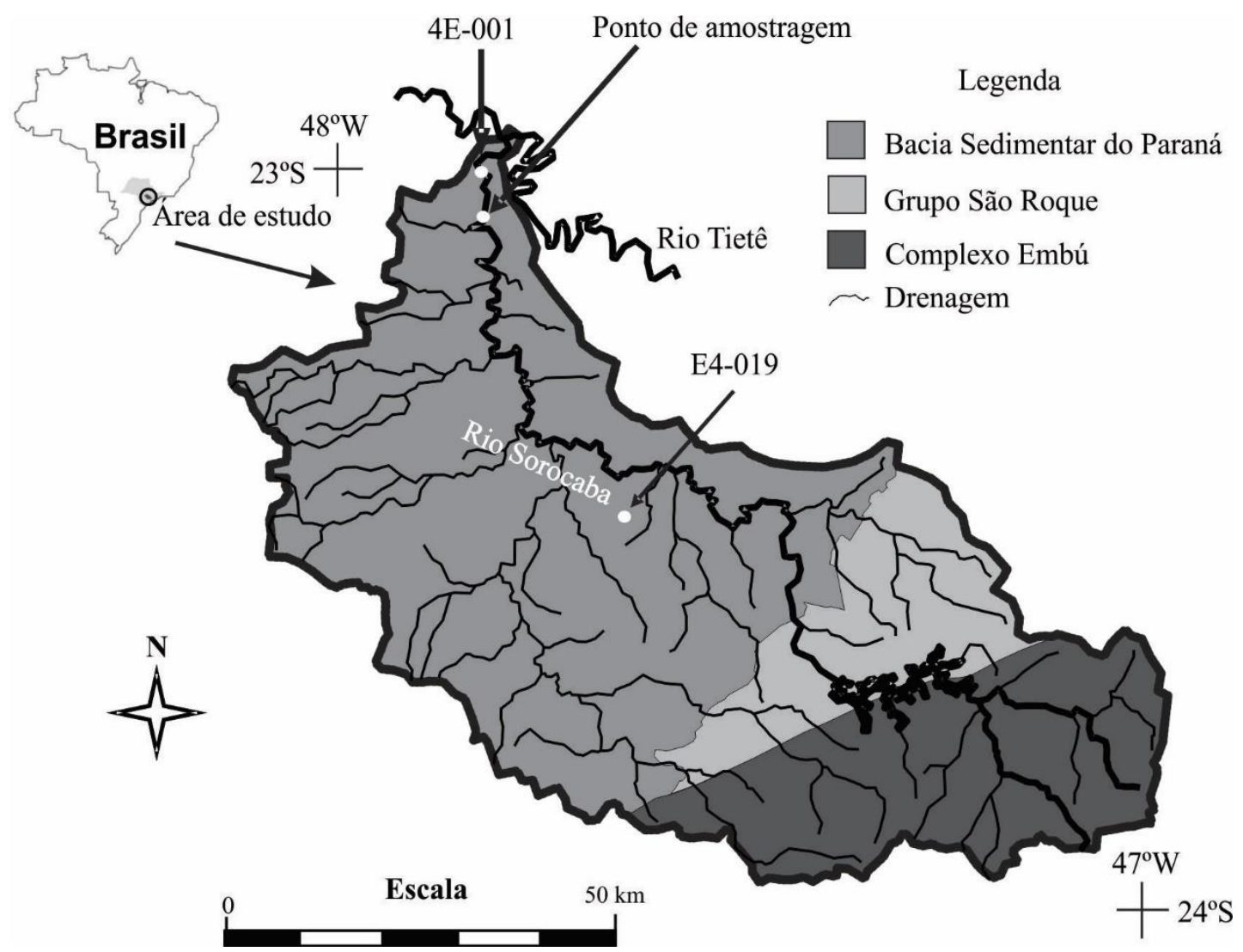

Figura 1. Bacia de drenagem do rio Sorocaba com a localização da estação de amostragem junto à foz, no município de Laranjal Paulista, e as estações fluviométrica (4E-001 - Entre Rios) e pluviométrica (E4-019 - Iperó).

A bacia do Rio Sorocaba está inserida em duas unidades geomorfológicas (Ross, 1996; Ross \& Moroz, 1997). Na porção leste encontrase a unidade Planaltos e Serras do Atlântico leste sudeste, que apresenta um maior grau de complexidade e tem sua gênese vinculada a vários ciclos de dobramentos acompanhados de metamorfismos regionais, falhamentos e extensas intrusões. O modelado dominante desta unidade é constituído por morros com formas de topos convexos, elevada densidade de canais de drenagem e vales profundos, apresentando altitudes entre 800 e 1000 metros e declividades acentuadas, acima de $20 \%$. As porções central e oeste da bacia do Rio Sorocaba situam-se na unidade Depressão Periférica da borda leste da
Bacia do Paraná, gerada por processos erosivos com alternância de ciclos secos e úmidos esculpida quase que totalmente nos sedimentos paleomesozóicos. Ela é constituída basicamente por colinas de topos amplos tabulares e convexos, onde predominam altitudes entre 600 e $700 \mathrm{~m}$ e declividades variando entre 5 e $10 \%$.

A geologia da área de estudo é constituída por rochas de idades variando desde o PréCambriano até o Cenozóico (IPT, 2006). No Embasamento Cristalino (Planaltos e Serras do Atlântico leste sudeste), destacam-se dois principais domínios litológicos. O Domínio São Roque apresenta rochas de baixo grau metamórfico do Grupo São Roque e complexos granitóides associados, tais como os granitos 
Sorocaba e São Roque. No Domínio Embu predomina paragnaisses e migmatitos associados ao Complexo Embu, ocorrendo também à presença dos granitos Ibiúna e Caucaia. Na Bacia Sedimentar do Paraná (Depressão Periférica da borda leste da Bacia do Paraná) destacam-se as rochas pertencentes aos grupos Itararé, Guatá e Passa Dois, as quais correspondem a diferentes ciclos deposicionais e são constituídos predominantemente por arenitos, siltitos e argilitos maciços ou estratificados, como também ritmitos, varvitos com seixos pingados e diamictitos.

O clima da região é caracterizado pelo predomínio de chuvas no verão e estiagem no inverno. Segundo Köppen (1948), é classificado como Cwa (clima temperado úmido com inverno seco e verão quente) na região da Depressão Periférica da borda leste da Bacia do
Paraná e Cwb (clima temperado úmido com inverno seco e verão temperado) na região Planaltos e Serras do Atlântico leste sudeste. A Figura 2 ilustra as médias mensais de vazão (a) e precipitação (b) para o período de 1984 a 2008, calculadas a partir de dados diários das estações fluviométrica 4E-001 Entre Rios (230.' S, $\left.47^{\circ} 48^{\prime} \mathrm{O}\right)$ e pluviométrica E4-019 Iperó ( $\left.23^{\circ} 20^{\prime} \mathrm{S}, 47^{\circ} 41^{\prime} \mathrm{O}\right)$ (DAEE/CTH, comunicação pessoal), localizadas junto à foz do Rio Sorocaba e na região central da bacia de drenagem, respectivamente (Figura 1). Para esse período de 25 anos, a vazão média anual foi de $63,1 \mathrm{~m}^{3} \mathrm{~s}^{-1}$, sendo fevereiro o mês de maior vazão média $\left(122,1 \mathrm{~m}^{3} \mathrm{~s}^{-1}\right)$ e agosto o de menor $\left(37,5 \mathrm{~m}^{3} \mathrm{~s}^{-1}\right)$. A precipitação média anual foi de $1243,1 \mathrm{~mm}$, sendo janeiro o mês mais chuvoso $(243,7 \mathrm{~mm}) \mathrm{e}$ agosto o mais seco $(27,2 \mathrm{~mm})$.

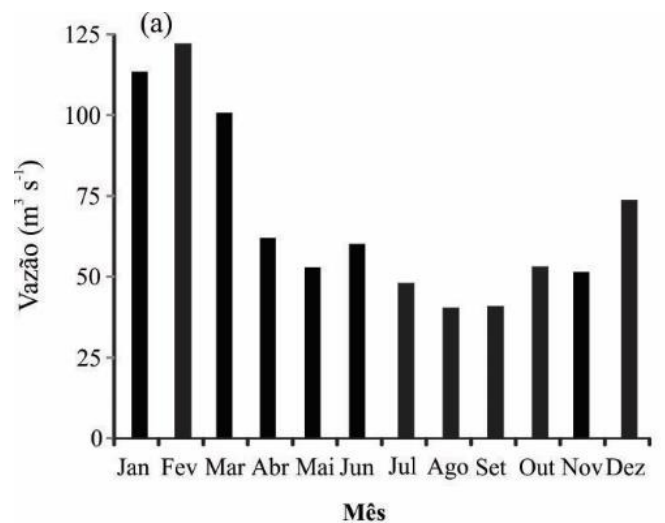

Figura 2. Médias mensais de Vazão (Q) e Precipitação (P) na bacia do rio Sorocaba para o período de 1984-2008.

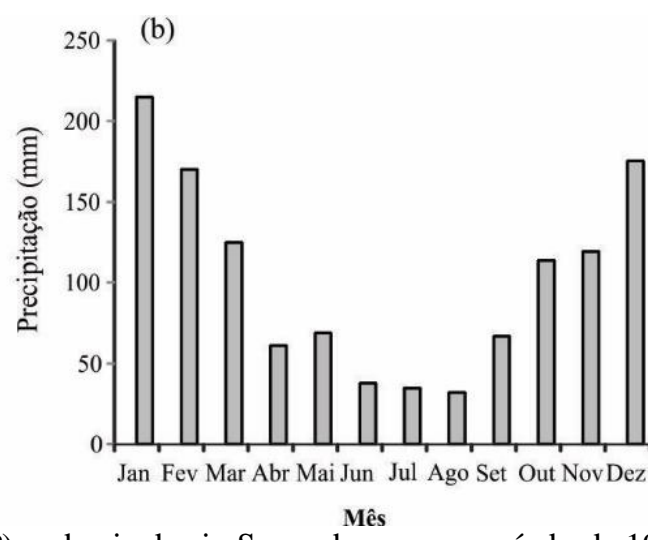

\section{DESENVOLVIMENTO METODOLÓGICO}

Foram realizadas 12 coletas de amostras de águas fluviais junto à foz do Rio Sorocaba, no município de Laranjal Paulista, no período de junho de 2009 a junho de 2010. A cada coleta foram amostrados $500 \mathrm{~mL}$ de águas fluviais nas margens esquerda e direita do rio e no eixo principal da corrente, local de maior fluxo de água, sempre a $1,5 \mathrm{~m}$ de profundidade, utilizando um amostrador pontual de estágio simples adaptado para rios de médio porte (Mortatti, 1995). Ainda em campo, as amostras foram compostas $(1,5 \mathrm{~L})$ e separadas em duas alíquotas de $500 \mathrm{~mL}$, uma bruta e outra preservada com $0,1 \mathrm{~mL}$ de $\mathrm{H}_{2} \mathrm{SO}_{4}$ concentrado, descartando-se o excedente. As amostras foram armazenadas em frascos de polietileno identificados e mantidas sob refrigeração a $4^{\circ} \mathrm{C}$ até processamento em laboratório.
Os parâmetros potencial hidrogeniônico $(\mathrm{pH})$, condutividade elétrica ( $\mathrm{CE}$, em $\mu \mathrm{S} \mathrm{cm}^{-1}$ ) e temperatura $\left(\mathrm{T}\right.$, em $\left.{ }^{\circ} \mathrm{C}\right)$ foram obtidos em campo, imediatamente após a coleta das amostras, utilizando os equipamentos portáteis Digimed DM 2 (pH) e Digimed DM4 (CE e T). Determinou-se a vazão instantânea do curso d'água $\left(\mathrm{Q}, \mathrm{em} \mathrm{m}^{3} \mathrm{~s}^{-1}\right)$ a cada amostragem, resultado do produto entre a área úmida da seção transversal do canal fluvial, caracterizada por batimetria, e a velocidade média do fluxo de água nessa seção, calculada a partir dos valores observados a $20 \%$ e $60 \%$ da altura da lâmina de água nas margens esquerda e direita e no eixo da corrente, com a utilização de um Micromolinete Digital Global Water FP 101.

As amostras de água fluvial bruta foram filtradas em filtro de membrana celulose 
"Millipore acetato" de 0,45 $\mu \mathrm{m}$ e analisada em Cromatógrafo Iônico Dionex ICS-90 equipado com colunas IonPac ${ }^{\circledR}$ CS12A 4x250 mm Analytical e IonPac ${ }^{\circledR}$ AS14A 4x250 mm Analytical, para a quantificação dos íons $\mathrm{Ca}^{2+}$, $\mathrm{Mg}^{2+}, \mathrm{Na}^{+}, \mathrm{K}^{+}, \mathrm{Cl}^{-}, \mathrm{SO}_{4}{ }^{2-}, \mathrm{NO}_{3}{ }^{-}$e $\mathrm{PO}_{4}{ }^{3-}$, com limites de detecção de $0,001 \mathrm{mg} \mathrm{L}^{-1}$ (Dionex Corporation, 2004) e de quantificação de 0,01 $\mathrm{mg} \mathrm{L}^{-1}$ (Ribani et al., 2004). Essas amostras brutas filtradas também foram utilizadas para a quantificação do $\mathrm{HCO}_{3}{ }^{-}$, representado pelos teores de alcalinidade obtidos pelo método da micro-titulação de Gran (Edmond, 1970). As amostras preservadas foram filtradas em filtro de fibra de vidro de 0,3-0,6 $\mu \mathrm{m}$ e analisadas por espectrometria de emissão ótica com plasma induzido de argônio acoplado indutivamente, utilizando o equipamento ICP-OES Optima $3000 \mathrm{DV}$, para determinar a concentração de Si, com limite de detecção de $0,02 \mathrm{mg} \mathrm{L}^{-1}$, sendo o resultado expresso em termos de $\mathrm{SiO}_{2}$.

As concentrações médias anuais normalizadas pela vazão de cátions e ânions foram calculadas de acordo com o procedimento estabelecido por Probst (1992), expresso na Equação 1. O transporte fluvial total foi quantificado utilizando a metodologia estocástica (Probst et al., 1992b), conforme Equação 2.

$$
\begin{aligned}
& C_{M N Q}=\frac{\sum_{i=1}^{n} C_{i} \cdot Q_{i}}{\sum_{i=1}^{n} Q_{i}} \\
& T=C_{M N Q} \times \bar{Q} \times f
\end{aligned}
$$

Onde:

$C_{M N Q}=$ concentração média normalizada pela vazão $\left(\mathrm{mg} \mathrm{L}^{-1}\right)$;

$C_{i}=$ concentração do íon na i-ésima amostra (mg $\left.\mathrm{L}^{-1}\right)$;

$Q_{i}=$ vazão instantânea medida no dia de coleta da i-ésima amostra $\left(\mathrm{m}^{3} \mathrm{~s}^{-1}\right)$;

$\bar{Q}=$ vazão média do período estudado $\left(\mathrm{m}^{3} \mathrm{~s}^{-1}\right)$;

$f=$ corresponde à correção da massa e do tempo, sendo igual a 31,536 para resultado em toneladas por ano.

\section{RESULTADOS E DISCUSSÃO}

\section{Hidroquímica}

Os parâmetros físicos e químicos analisados nas águas do Rio Sorocaba podem ser observados na Tabela 1, onde o período seco compreende os meses de junho a setembro de 2009 e abril a junho de 2010 e o período chuvoso os meses de outubro de 2009 a março de 2010.

A vazão apresentou variação sazonal marcante, com menores valores no período seco (média de 70,73 $\mathrm{m}^{3} \mathrm{~s}^{-1}$ ) e maiores no período chuvoso (média de 222,50 $\mathrm{m}^{3} \quad \mathrm{~s}^{-1}$ ), acompanhando a sazonalidade verificada na distribuição das médias mensais da série histórica (Figura 2b). Apesar dessa similaridade, a vazão média anual calculada para o período de estudo, considerando as vazões instantâneas de cada amostragem $\left(146,62 \mathrm{~m}^{3} \mathrm{~s}^{-1}\right)$, mostrou-se 2,3 vezes maior que a média histórica de 25 anos $\left(63,1 \mathrm{~m}^{3} \mathrm{~s}^{-1}\right)$.

A variação de $\mathrm{pH}$ entre 6,9 e 7,3 mostrou que as águas do Rio Sorocaba encontram-se próxima à neutralidade. Situação semelhante às observadas para os rios Tietê $(6,9$ a 7,4$)$, no município de Tietê - SP, e Piracicaba $(6,8$ a 7,4$)$, no município de Artêmis-SP, respectivamente (Bortoletto Junior, 2004), rios que junto com o Sorocaba compõem a bacia do Médio Tietê. A condutividade elétrica (CE) apresentou variabilidade sazonal expressiva, com mínimo de $76,00 \mu \mathrm{S} \mathrm{cm}^{-1}$ em dezembro de 2009 , durante o período chuvoso, e máximo de $149,10 \mu \mathrm{S} \mathrm{cm}^{-}$ 1 em junho de 2010, no período seco, acima do limite superior esperado para águas naturais, de $100 \mu \mathrm{S} \mathrm{cm}^{-1}$ (Hermes \& Silva, 2004). Os valores de CE mostraram-se inferiores aos observados para os rios Tietê (de 254 a $697 \mu \mathrm{S} \mathrm{cm} \mathrm{cm}^{-1}$ ) e Piracicaba (111 a $\left.455 \mu \mathrm{S} \mathrm{cm} \mathrm{cm}^{-1}\right)$. A temperatura da água variou entre $15,6^{\circ} \mathrm{C}$ e $33,0^{\circ} \mathrm{C}$ e acompanhou a sazonalidade climática da região, com mínima observada em junho de 2009 (inverno) e máximo em janeiro de 2010 (verão). 
Tabela 1. Parâmetros físicos e químicos das águas do rio Sorocaba junto à foz, durante o período de estudo.

\begin{tabular}{|c|c|c|c|c|c|c|c|c|c|c|c|c|c|c|c|}
\hline $\begin{array}{c}\begin{array}{c}\text { Data de } \\
\text { amostragem }\end{array} \\
\end{array}$ & $\begin{array}{c}\mathrm{Q}^{(\mathrm{a})} \\
\left(\mathrm{m}^{3} \mathrm{~s}^{-1}\right) \\
\end{array}$ & $\mathrm{pH}$ & $\begin{array}{c}\mathrm{CE}^{(\mathrm{b})} \\
\left(\mu \mathrm{S} \mathrm{cm}^{-1}\right)\end{array}$ & $\begin{array}{l}\mathrm{T}^{(\mathrm{c})} \\
\left({ }^{\circ} \mathrm{C}\right) \\
\end{array}$ & \multicolumn{11}{|c|}{$\left(\mathrm{mg} \mathrm{L}^{-1}\right)$} \\
\hline $17 / 06 / 2009$ & 37,47 & 6,9 & 143,20 & 15,60 & 35,90 & 16,87 & 1,52 & 27,18 & 1,82 & 47,52 & 17,20 & 9,95 & 10,55 & 0,60 & 169,11 \\
\hline $21 / 07 / 2009$ & 43,71 & 6,9 & 123,90 & 16,90 & 31,00 & 16,36 & 1,47 & 23,67 & 1,70 & 42,00 & 16,30 & 9,10 & 8,88 & 0,47 & 150,95 \\
\hline $25 / 08 / 2009$ & 123,23 & 6,9 & 109,00 & 16,90 & 14,12 & 11,75 & 1,08 & 10,61 & 1,45 & 34,66 & 8,48 & 5,05 & 2,74 & 0,15 & 90,08 \\
\hline $22 / 09 / 2009$ & 99,84 & 7,1 & 121,80 & 20,30 & 17,50 & 13,54 & 1,05 & 11,16 & 1,47 & 42,30 & 8,54 & 5,36 & 3,26 & 0,12 & 104,29 \\
\hline $10 / 11 / 2009$ & 170,01 & 6,9 & 108,10 & 26,40 & 11,89 & 10,80 & 1,00 & 10,20 & 1,40 & 34,40 & 8,19 & 5,40 & 2,78 & $<0,01$ & 86,06 \\
\hline $12 / 12 / 2009$ & 363,35 & 6,9 & 76,00 & 25,70 & 10,43 & 8,40 & 0,84 & 5,70 & 1,00 & 26,73 & 4,20 & 4,80 & 0,93 & 0,09 & 63,12 \\
\hline $20 / 01 / 2010$ & 366,47 & 7,1 & 79,70 & 33,00 & 10,44 & 8,50 & 0,82 & 6,03 & 1,10 & 30,95 & 4,33 & 3,23 & 0,96 & $<0,01$ & 66,35 \\
\hline $23 / 02 / 2010$ & 184,04 & 7,1 & 99,60 & 29,00 & 12,45 & 10,50 & 1,20 & 7,70 & 1,70 & 39,20 & 5,87 & 3,36 & 3,96 & $<0,01$ & 85,94 \\
\hline $30 / 03 / 2010$ & 151,30 & 7,3 & 87,10 & 26,0 & 11,49 & 11,11 & 1,25 & 7,70 & 1,70 & 31,94 & 5,95 & 4,40 & 4,50 & 0,22 & 80,25 \\
\hline $27 / 04 / 2010$ & 107,64 & 7,1 & 103,60 & 25,70 & 12,21 & 13,35 & 1,30 & 9,14 & 1,80 & 40,28 & 7,02 & 5,04 & 5,98 & 0,11 & 96,23 \\
\hline $27 / 05 / 2010$ & 70,21 & 7,1 & 128,60 & 22,20 & 22,00 & 13,26 & 1,40 & 14,92 & 1,90 & 41,08 & 11,27 & 6,22 & 8,25 & 0,33 & 120,61 \\
\hline $24 / 06 / 2010$ & 42,15 & 7,3 & 149,10 & 21,10 & 29,00 & 13,69 & 1,90 & 18,83 & 2,10 & 46,34 & 12,23 & 10,34 & 10,14 & 0,37 & 144,94 \\
\hline \multicolumn{16}{|c|}{ Período de estudo } \\
\hline Média $^{(e)}$ & 146,62 & 7,0 & 110,81 & 23,30 & 13,61 & 10,63 & 1,06 & 9,03 & 1,40 & 34,24 & 6,71 & 4,82 & 3,22 & 0,18 & 84,82 \\
\hline $\mathrm{DP}^{(\mathrm{f})}$ & 113,13 & 0,2 & 23,46 & 5,36 & 0,14 & 0,05 & 0,01 & 0,11 & 0,01 & 0,14 & 0,07 & 0,04 & 0,06 & 0,01 & 0,58 \\
\hline \multicolumn{16}{|c|}{ Período seco } \\
\hline Média $^{(\mathrm{e})}$ & 70,73 & 7,0 & 126,23 & 19,37 & 20,08 & 13,52 & 1,35 & 14,57 & 1,74 & 40,20 & 10,52 & 6,62 & 6,53 & 0,26 & 115,39 \\
\hline $\mathrm{DP}^{(\mathrm{f})}$ & 36,80 & 0,2 & 18,06 & 3,92 & 0,40 & 0,08 & 0,01 & 0,29 & 0,01 & 0,21 & 0,17 & 0,10 & 0,14 & 0,01 & 1,34 \\
\hline \multicolumn{16}{|c|}{ Período chuvoso } \\
\hline Média $^{(\mathrm{e})}$ & 222,50 & 7,0 & 95,38 & 26,87 & 11,55 & 9,71 & 0,97 & 7,27 & 1,29 & 32,34 & 5,49 & 4,24 & 2,17 & 0,13 & 75,10 \\
\hline $\mathrm{DP}^{(\mathrm{f})}$ & 113,94 & 0,2 & 17,71 & 4,17 & 0,05 & 0,04 & 0,01 & 0,05 & 0,01 & 0,13 & 0,04 & 0,02 & 0,04 & 0,01 & 0,34 \\
\hline
\end{tabular}

As concentrações das espécies químicas estudadas mostraram-se inversamente relacionadas com a variação das vazões instantâneas, em maior ou menor grau, sendo as menores concentrações verificadas no período de maiores vazões, de novembro 2009 a março 2010, e vice-versa. Destaca-se que durante o período estudado, as maiores concentrações médias normalizadas pela vazão foram de $\mathrm{Ca}^{2+}$ $\left(10,63 \mathrm{mg} \mathrm{L}^{-1}\right) \mathrm{e} \mathrm{Na}^{+}\left(9,03 \mathrm{mg} \mathrm{L}^{-1}\right)$ entre os cátions e de $\mathrm{HCO}_{3}^{-}\left(34,24 \mathrm{mg} \mathrm{L}^{-1}\right) \mathrm{e} \mathrm{Cl}^{-}(6,71 \mathrm{mg}$ $\left.\mathrm{L}^{-1}\right)$ entre os ânions, além da presença significativa de $\mathrm{SiO}_{2}\left(13,61 \mathrm{mg} \mathrm{L}^{-1}\right)$. Para as demais espécies químicas, as concentrações médias foram inferiores a $5,00 \mathrm{mg} \mathrm{L}^{-1}$. Nos períodos seco e chuvoso observou-se comportamento semelhante, exceto entre os cátions, onde a concentração média de $\mathrm{Na}^{+}$ $\left(14,57 \mathrm{mg} \mathrm{L}^{-1}\right)$ foi superior à de $\mathrm{Ca}^{2+}(13,52 \mathrm{mg}$ $\left.\mathrm{L}^{-1}\right)$.

A partir das concentrações médias observadas na Tabela 1 , foi estabelecida a distribuição percentual das principais espécies químicas dissolvidas em relação ao TDS, considerando sua concentração molar (Peray, 1998), ilustrado na Figura 3. A espécie química de maior contribuição ao TDS foi o $\mathrm{HCO}_{3}^{-}$ $(30,8 \%)$, seguido por $\mathrm{Na}^{+}(21,6 \%), \mathrm{Ca}^{2+}$
(14,6\%), $\mathrm{SiO}_{2}(12,5 \%)$ e $\mathrm{Cl}^{-}(10,4 \%)$, que juntas representaram $90 \%$ do TDS presente no Rio Sorocaba. A participação de $\mathrm{HCO}_{3}{ }^{-}$no TDS se mostrou inferior à observada no Rio Tietê $(40,4 \%)$ e na mesma proporção que no Rio Piracicaba $(30,2 \%)$, enquanto que para o $\mathrm{Na}^{+}$, a participação no Rio Sorocaba foi superior às verificadas nos rios Tietê $(14,0 \%)$ e Piracicaba $(16,1 \%)$ (Bortoletto Junior, 2004).

Considerando um pressuposto equilíbrio iônico para as águas naturais, expresso pela razão ente o somatório de cátions e de ânions $\left(\Sigma^{+} / \Sigma^{-}=1\right.$, em $\mu$ eq $L^{-1}$ ) (Probst, 1992), foi estabelecido o balanço de cargas para as espécies químicas dissolvidas no Rio Sorocaba (Figura 4). Observou-se um déficit de cargas aniônicas, esperado para amostras fluviais pouco mineralizadas e que pode ser atribuído à presença de ânions orgânicos dissolvidos não contabilizados (Tardy et al., 2005), como o carbono orgânico dissolvido, que apresenta concentrações na ordem de 5,35 $\mu$ eq por mg de C (Drever, 1997). Tal déficit é relatado na literatura para outras bacias de drenagem, como por exemplo, as dos rios Congo e Ubangui (Probst et al., 1992a), Níger (Boeglin \& Probst, 1996), Tietê e Piracicaba (Bortoletto Junior, 
2004), Amazonas (Tardy et al., 2005), Jundiaí e

Capivari (Oliveira et al., 2014).

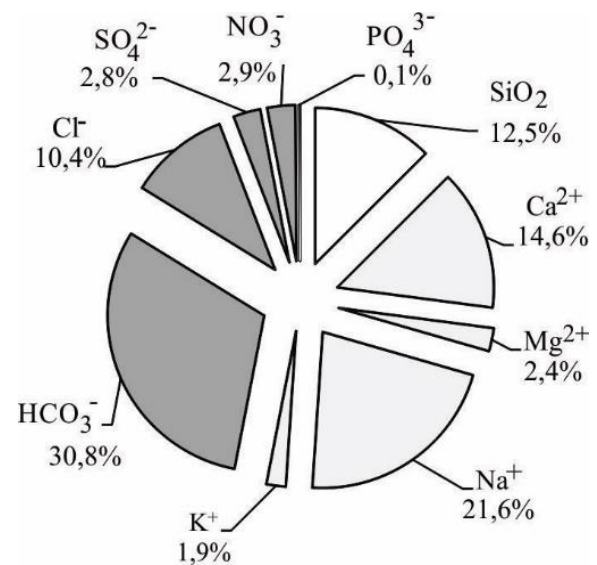

Figura 3. Partição percentual das principais espécies químicas presentes nas amostras de águas do rio Sorocaba junto à foz em Laranjal Paulista, SP, durante o período estudado.

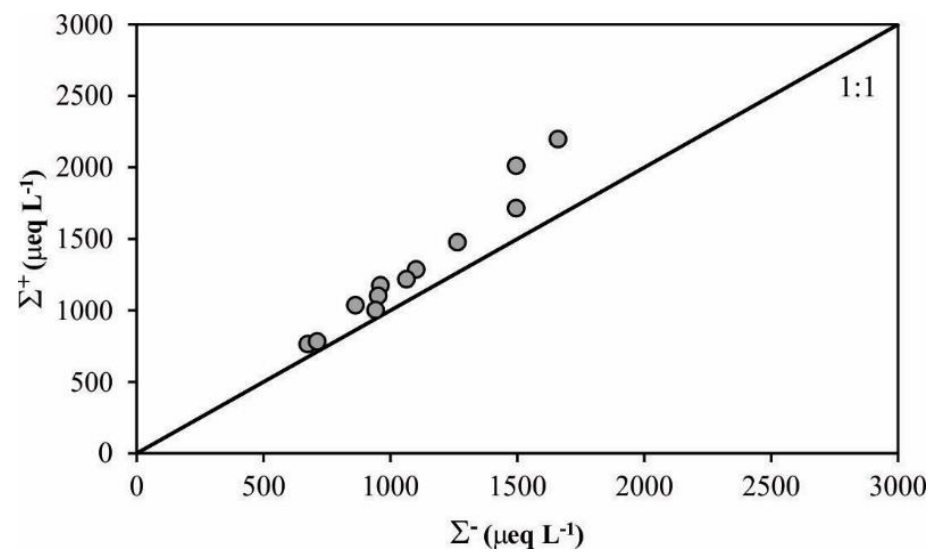

Figura 4. Balanço de cargas das principais espécies químicas dissolvidas no rio Sorocaba, no período estudado, obtido pela relação entre o somatório de cátions $\left(\Sigma^{+}\right)$e o somatório de ânions $\left(\Sigma^{-}\right)$.

\section{Relação vazão $x$ concentração}

O estudo das relações concentração x vazão é um importante parâmetro na caracterização hidroquímica fluvial, pois em condições naturais a composição química das águas fluviais mostrase variável ao longo do tempo em função do regime hidrológico e da atividade biológica na bacia hidrográfica (Meybeck, 1986). A aplicação de modelos de regressão a essas relações permite identificar possíveis origens das espécies químicas e caracterizar alguns dos mecanismos susceptíveis de influenciar ou controlar o comportamento das espécies químicas dissolvidas nas águas fluviais. Hall $(1970$, 1971) testou diversos modelos na tentativa de explicar a evolução das concentrações das espécies químicas dissolvidas com respeito às vazões na identificação de aportes pontuais e difusos.

A utilização de um modelo de potência $(C=$ a. $\left.Q^{b}\right)$ possibilita avaliar o comportamento das espécies químicas dissolvidas em função da vazão (Kattan \& Probst, 1986; Mortatti et al., 2008, Oliveira et al., 2014). A comparação deste modelo de potencia com um modelo de curva de diluição teórica das espécies químicas de interesse, o qual representa o processo de concentração e diluição pontual infinita a partir de uma concentração inicial (Hall, 1971) e é expresso pela Equação 3 (Kattan \& Probst, 1986; Probst et al., 1992a, Mortatti, 1995; Mortatti et al., 2008, Oliveira et al., 2014), permite identificar as possíveis origens dessas espécies químicas em dois grupos principais, aportes pontuais e difusos. $\mathrm{O}$ primeiro caracteriza-se pela proximidade ou mesmo sobreposição das curvas de regressão e de diluição teórica obtidas, sendo relacionado principalmente a aportes de origem antrópica, como por exemplo, o lançamento de esgotos domésticos in natura e de efluentes industriais nos corpos hídricos. Enquanto que o segundo 
apresenta um evidente distanciamento entre essas curvas e os aportes são associados tanto a uma origem natural quanto antrópica ao longo da bacia de drenagem, tais como os processos de alteração de rochas, os aportes atmosféricos e ao manejo de áreas agrícolas (Mortatti et al., 2008).

$C_{i}=\frac{C_{\max } \cdot Q_{\min }}{Q_{i}}$

Onde:

$C_{i}=$ concentração da espécie química na curva de diluição;
$C_{\max }=$ concentração máxima observada da espécie química;

$Q_{\min }=$ vazão mínima observada;

$Q_{i}=$ vazão do dia de amostragem.

As relações concentração $\mathrm{x}$ vazão obtidas para as espécies químicas dissolvidas e o TDS junto à foz do Rio Sorocaba mostraram-se decrescentes e influenciadas pela diluição fluvial, e apresentaram modelos de potência ajustados aos dados altamente significativos $(p<$ 0,01) (Figura 5).
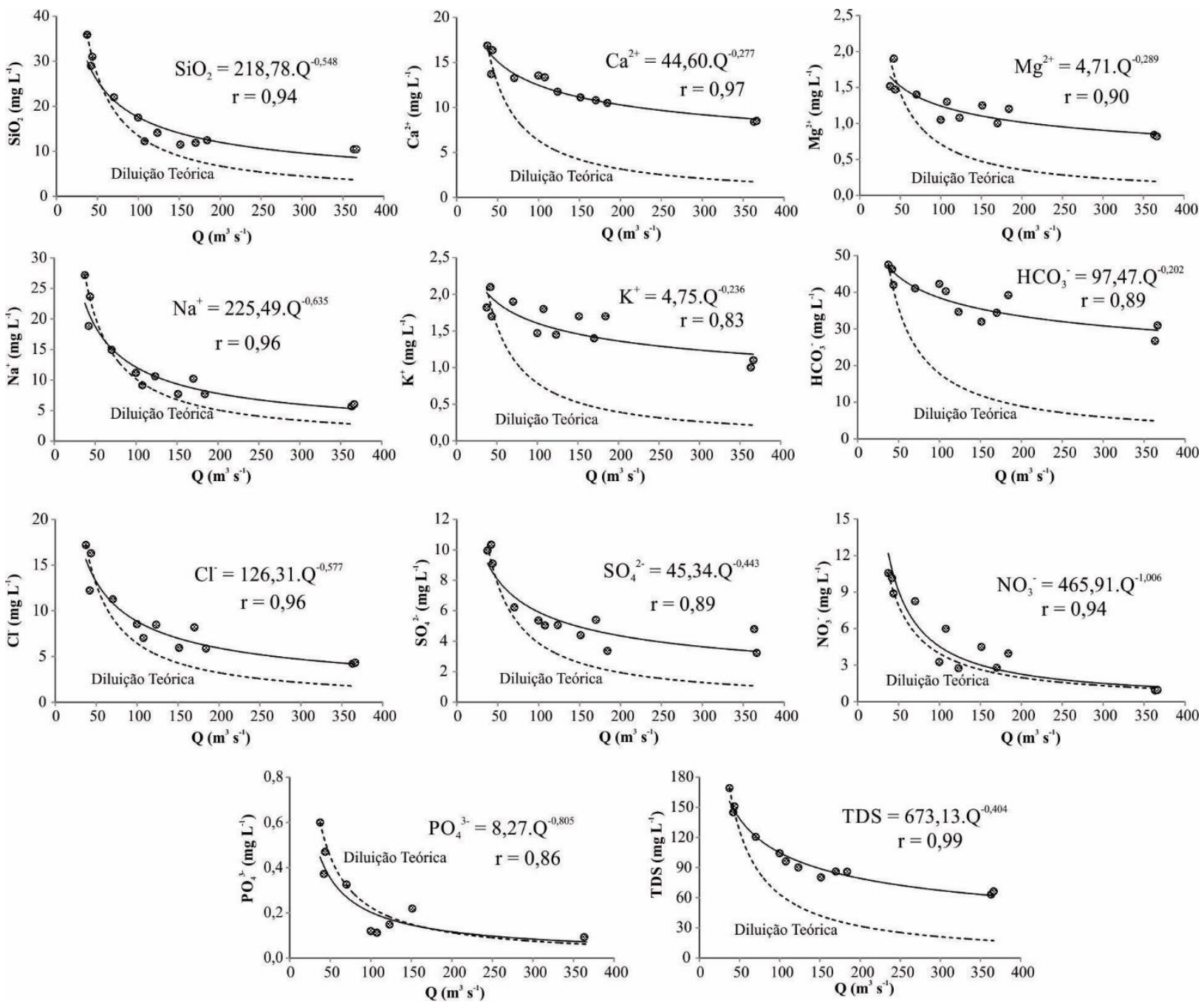

Figura 5. Relação concentração x vazão para as principais espécies químicas dissolvidas e TDS no rio Sorocaba junto à foz, com as respectivas regressões de potência e curvas de diluição teórica, durante o período estudado.

A comparação dos modelos de potência obtidos para $\mathrm{Ca}^{2+}, \mathrm{Mg}^{2+}, \mathrm{K}^{+}, \mathrm{HCO}_{3}{ }^{-}$e TDS com as respectivas curvas de diluição teórica indicaram predomínio de aportes difusos, dado o aparente distanciamento entre as curvas. Já a proximidade entre as curvas obtidas para $\mathrm{Na}^{+}$, $\mathrm{NO}_{3}{ }^{-}$e $\mathrm{PO}_{4}{ }^{3-}$ apontam uma associação com aportes pontuais. Para o $\mathrm{SiO}_{2}, \mathrm{Cl}^{-}$e $\mathrm{SO}_{4}{ }^{2-}$ não foi possível identificar claramente os aportes predominantes pela comparação do posicionamento das curvas, podendo inferir uma mistura de ambas as fontes.

Assim, propõe-se que a avaliação da possível origem das espécies químicas dissolvidas, pontual ou difusa, também pode ser interpretada pelo valor do expoente $b$ na função do modelo 
ajustado aos dados em comparação com o obtido para a curva de diluição teórica, que apresenta valor -1,0. Quanto mais próximo a $-1,0$ for o expoente $b$ na função do modelo ajustado aos dados, o predomínio será dos aportes pontuais. Para $b$ na função do modelo ajustado aos dados mais próximos à zero, o predomínio será dos aportes difusos. A Figura 5 ilustra essas situações, com $\mathrm{Ca}^{2+}, \mathrm{Mg}^{2+}, \mathrm{K}^{+}, \mathrm{HCO}_{3}^{-}$e TDS apresentando expoentes $b$ maiores que $-0,40 \mathrm{e}$ relacionados a aportes de origem difusa; enquanto que os valores de $b$ menores que $-0,60$ observados para $\mathrm{Na}^{+}, \mathrm{NO}_{3}{ }^{-}$e $\mathrm{PO}_{4}{ }^{3-}$ indicam uma associação com aportes pontuais. Para valores de $b$ próximos a $-0,50$ se faz necessária uma interpretação mais cuidadosa, como o observado para $\mathrm{SiO}_{2}, \mathrm{Cl}^{-}$e $\mathrm{SO}_{4}{ }^{2-}$, indicando a mistura das fontes, confirmando $\mathrm{o}$ fato descrito anteriormente.

\section{Transporte fluvial}

O total de sólidos dissolvidos transportados pelo Rio Sorocaba foi de $392,16 \times 10^{3} \mathrm{t} \mathrm{a}^{-1}$, carga que corresponde à contribuição para a bacia do Médio Tietê no período de estudo. Entre as espécies químicas analisadas, as maiores cargas transportadas foram de $\mathrm{HCO}_{3}^{-}$e $\mathrm{SiO}_{2}$, com $158,30 \times 10^{3}$ e $62,91 \times 10^{3} \mathrm{ta}^{-1}$, respectivamente, seguidas de $\mathrm{Ca}^{2+}, \mathrm{Na}^{+}, \mathrm{Cl}^{-}, \mathrm{SO}_{4}{ }^{2-}, \mathrm{NO}_{3}{ }^{-}, \mathrm{K}^{+}$, $\mathrm{Mg}^{2+}$ e $\mathrm{PO}_{4}{ }^{3-}$ (Tabela 2). Para os períodos seco e chuvoso observou-se distribuição semelhante, com maiores cargas de $\mathrm{HCO}_{3}{ }^{-}$e $\mathrm{SiO}_{2}$, seguidas pelas demais espécies químicas; com exceção do maior transporte de $\mathrm{Na}^{+}$em relação ao $\mathrm{Ca}^{2+}$ no período seco. Destaca-se ainda que o $\mathrm{NO}_{3}{ }^{-}$não apresentou variação sazonal significativa, com uma carga transportada na ordem de 14,89 x $10^{3}$ $\mathrm{t} \mathrm{a}^{-1}$. Verificou-se um aumento expressivo dos transportes no período chuvoso em relação ao período seco para todos as espécies químicas quantificadas neste estudo. Para o $\mathrm{HCO}_{3}{ }^{-}$esse aumento foi de cerca de 2,5 vezes; para o $\mathrm{Ca}^{2+}$, $\mathrm{Mg}^{2+} \mathrm{e} \mathrm{K}^{+}$, na ordem de 2,3 vezes; e, para o $\mathrm{SO}_{4}{ }^{2-}$ em 2 vezes.

Tabela 2. Transporte total de $\mathrm{SiO}_{2}$, cátions, ânions e do total de sólidos dissolvidos presentes nas águas do rio Sorocaba junto à foz, durante o período de estudo.

\begin{tabular}{lcccccccccccc}
\hline Período & $\mathrm{SiO}_{2}$ & $\mathrm{Ca}^{2+}$ & $\mathrm{Mg}^{2+}$ & $\mathrm{Na}^{+}$ & $\mathrm{K}^{+}$ & $\begin{array}{l}\mathrm{HCO}_{3}^{-} \\
\left(10^{3} \mathrm{ta}^{-1}\right)\end{array}$ & $\mathrm{Cl}^{-}$ & $\mathrm{SO}_{4}{ }^{2-}$ & $\mathrm{NO}_{3}^{-}$ & $\mathrm{PO}_{4}{ }^{3-}$ & $\mathrm{TDS}$ \\
\hline Estudo & 62,91 & 49,17 & 4,90 & 41,77 & 6,46 & 158,30 & 31,01 & 22,27 & 14,89 & 0,85 & 392,16 \\
Seco & 44,78 & 30,17 & 3,01 & 32,51 & 3,87 & 89,67 & 23,43 & 14,76 & 14,57 & 0,59 & 257,39 \\
Chuvoso & 81,03 & 68,16 & 6,78 & 51,03 & 9,05 & 226,92 & 38,55 & 29,77 & 15,22 & 0,90 & 526,93 \\
\hline
\end{tabular}

O transporte específico de $\mathrm{SiO}_{2}$, cátions, ânions e TDS, obtido através da ponderação do transporte total anual pela área de contribuição da bacia de drenagem, permitiu a comparação das cargas específicas transportadas pelo Rio
Sorocaba com as apresentadas por Bortoletto Junior (2004) para os rios Tietê e Piracicaba, nos municípios de Tietê e Artemis, respectivamente (Tabela 3).

Tabela 3. Transporte específico de $\mathrm{SiO}_{2}$, cátions, ânions e do total de sólidos dissolvidos para os rios Sorocaba, Tietê e Piracicaba.

\begin{tabular}{cccccccccccc}
\hline Rio & $\mathrm{SiO}_{2}$ & $\mathrm{Ca}^{2+}$ & $\mathrm{Mg}^{2+}$ & $\mathrm{Na}^{+}$ & $\mathrm{K}^{+}$ & $\begin{array}{c}\mathrm{HCO}_{3}^{-} \\
\left(\mathrm{t} \mathrm{km}^{-2} \mathrm{a}^{-1}\right)\end{array}$ & $\mathrm{Cl}^{-}$ & $\mathrm{SO}_{4}^{2-}$ & $\mathrm{NO}_{3}^{-}$ & $\mathrm{PO}_{4}{ }^{3-}$ & $\mathrm{TDS}^{2}$ \\
\hline Sorocaba & 11,9 & 9,3 & 0,9 & 7,9 & 1,2 & 30,0 & 5,9 & 4,2 & 2,8 & 0,2 & 74,4 \\
Tietê $^{(1)}$ & 5,7 & 10,0 & 2,0 & 21,1 & 5,2 & 61,5 & 23,8 & 14,6 & 0,3 & 1,3 & 151,7 \\
Piracicaba $^{(1)}$ & 3,6 & 3,3 & 1,2 & 8,1 & 1,4 & 15,2 & 7,3 & 9,5 & 0,2 & 0,1 & 50,3 \\
\hline
\end{tabular}

(1) Bortoletto Junior (2004), referente aos rios Tietê e Piracicaba nos municípios de Tietê e Artemis, respectivamente.

De maneira geral, os maiores transportes específicos foram observados para o Rio Tietê e uma alternância de valores intermediários entre os rios Sorocaba e Piracicaba. O transporte específico de TDS pelo Rio Sorocaba $(74,4$ t km
${ }^{2} \mathrm{a}^{-1}$ ) foi cerca de 2 vezes menor que o observado no Rio Tietê $\left(151,7 \mathrm{t} \mathrm{km}^{-2} \mathrm{a}^{-1}\right)$ e $50 \%$ maior que o do Rio Piracicaba $\left(50,3 \mathrm{t} \mathrm{km}^{-2} \mathrm{a}^{-1}\right)$. Em relação

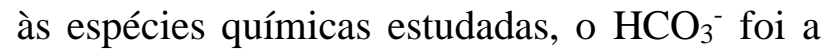
que apresentou o maior transporte específico e, 
comparativamente, apresentou as mesmas proporções observadas para o TDS. Para as demais espécies químicas analisadas não foi possível verificar o mesmo padrão.

As cargas específicas de $\mathrm{SiO}_{2}$ e $\mathrm{NO}_{3}{ }^{-}(11,9$ e $2,8 \mathrm{t} \mathrm{km}^{-2} \mathrm{a}^{-1}$, respectivamente) mostraram-se superiores aos outros dois rios, indicando que a bacia do Rio Sorocaba está sujeita a processos intempéricos de silicatos possivelmente mais intensos que as outras bacias e que a carga fluvial de $\mathrm{NO}_{3}^{-}$mostra-se mais sensível às influências das atividades humanas, relacionadas a fontes pontuais como $\mathrm{o}$ lançamento de esgotos domésticos sem tratamento nos corpos de água. O transporte específico de $\mathrm{Ca}^{2+}\left(2,8 \mathrm{t} \mathrm{km}^{-2} \mathrm{a}^{-1}\right)$ foi muito próximo ao observado no Rio Tietê e cerca de 3 vezes maior que o do Rio Piracicaba. Para o $\mathrm{Mg}^{2+}, \mathrm{Na}^{+}, \mathrm{K}^{+}$e $\mathrm{PO}_{4}{ }^{3-}$, as cargas específicas dos rios Sorocaba e Piracicaba foram da mesma ordem de grandeza. Os menores transportes específicos de $\mathrm{Cl}^{-}$e $\mathrm{SO}_{4}{ }^{2-}$ foram os do Rio Sorocaba. Essas diferenças ente cargas transportadas podem estar associadas, em parte, às pressões antrópicas a que as bacias estão sujeitas, como, por exemplo, distintos usos e ocupação do terreno e de aportes de efluentes domésticos sem tratamento prévio, entre outros.

\section{CONCLUSÕES}

A análise dos parâmetros físicos e químicos obtidos mostraram uma variação sazonal marcante da vazão, com menores volumes relacionados ao período seco e maiores ao período chuvoso, acompanhando a distribuição das médias mensais calculadas para 25 anos. $\mathrm{O}$ $\mathrm{pH}$ mostrou-se próximo à neutralidade, sem variação sazonal ao longo do período de estudo. A condutividade elétrica, influenciada pela variação da vazão, apresentou maiores concentrações no período seco, que foram superiores ao esperado para águas naturais, um indicativo da influência antrópica. A variação da temperatura das águas fluviais acompanhou a sazonalidade climática da região, com máxima em janeiro (verão).

As concentrações das espécies químicas se mostraram inversamente relacionadas com a variação das vazões, com um predomínio, em ordem de importância, de $\mathrm{HCO}_{3}{ }^{-}>\mathrm{Na}^{+}>\mathrm{Ca}^{2+}>$ $\mathrm{SiO}_{2}>\mathrm{Cl}^{-}$, que juntas representaram $90 \%$ do TDS presente no Rio Sorocaba. O balanço de cargas apresentou um déficit de ânions devido às baixas concentrações verificadas e à não inclusão de componentes orgânicos dissolvidos em seu cálculo, principalmente carbono orgânico dissolvido.

As relações concentração x vazão obtidas para as espécies químicas dissolvidas e o TDS mostraram-se decrescentes, confirmando a influência dos processos de diluição fluvial, e permitiram o ajuste significativo de modelos de potência. A análise comparativa desses modelos de regressão com os respectivos modelos de curvas de diluição teórica possibilitou verificar a associação de $\mathrm{Ca}^{2+}, \mathrm{Mg}^{2+}, \mathrm{K}^{+}, \mathrm{HCO}_{3}{ }^{-}$e $\mathrm{TDS}$ a aportes difusos e de $\mathrm{Na}^{+}, \mathrm{NO}_{3}{ }^{-}$e $\mathrm{PO}_{4}{ }^{3-}$ a aportes pontuais, não sendo possível identificar os aportes predominantes de $\mathrm{SiO}_{2}, \mathrm{Cl}^{-} \mathrm{e} \mathrm{SO}_{4}{ }^{2-}$. A avaliação proposta, com a comparação do expoente $b$ da função do modelo ajustado aos dados em relação ao da curva de diluição teórica mostrou-se válida.

No período estudado a contribuição do Rio Sorocaba para a calha do Médio Tietê, em termos de carga dissolvida, foi de $392,16 \times 10^{3} \mathrm{t}$ $\mathrm{a}^{-1}$, sendo as mais significativas as de $\mathrm{HCO}_{3}{ }^{-} \mathrm{e}$ $\mathrm{SiO}_{2}, \operatorname{com} 158,30 \times 10^{3}$ e $62,91 \times 10^{3} \mathrm{t} \mathrm{a}^{-1}$, respectivamente, seguidas de $\mathrm{Ca}^{2+}, \mathrm{Na}^{+}, \mathrm{Cl}^{-}$, $\mathrm{SO}_{4}{ }^{2-}, \mathrm{NO}_{3}{ }^{-}, \mathrm{K}^{+}, \mathrm{Mg}^{2+}$ e $\mathrm{PO}_{4}{ }^{3-}$. Houve um maior transporte no período chuvoso, indicando uma variação sazonal. A comparação das cargas específicas transportadas pelo Rio Sorocaba com as verificadas na literatura para os rios Tietê e Piracicaba indicou, de forma geral, maiores transportes no Rio Tietê e alternância de valores intermediários entre os rios Sorocaba e Piracicaba. Nos três rios, o maior transporte específico foi de $\mathrm{HCO}_{3}{ }^{-}$, enquanto que para as demais espécies químicas não foi verificado um padrão, com alternância de contribuição específica das espécies químicas, refletindo, em parte, diferenças nas pressões antrópicas a que cada bacia está sujeita.

A avaliação hidroquímica da carga dissolvida e de seu comportamento sazonal permitiu um melhor conhecimento do Rio Sorocaba, fornecendo subsídios para o gerenciamento desta importante bacia hidrográfica regional, com a adoção de planejamento e manejo 
adequados que contemplem um desenvolvimento econômico com equidade social e em harmonia com o meio ambiente, face às pressões antrópicas a que está sujeita, com vistas à manutenção e melhoria da qualidade das águas do Rio Sorocaba e de seus tributários, fundamental para a integridade do sistema.

\section{AGRADECIMENTOS}

Os autores agradecem à FAPESP (Processos no 08/57104-4 e 08/09369-9), ao CNPq (Processo ${ }^{\circ}$ 134169/2009-3) e à CAPES pelo apoio financeiro concedido, e à UNESP e ao CENA/USP que possibilitaram a realização da pesquisa.

\section{REFERÊNCIAS BIBLIOGRÁFICAS}

1. BARTH, T.F.W. Abundance of the elements, areal averages and geochemical cycles. Geochimimica et Cosmochimica Acta, v.23, p.1-8, 1961.

2. BOEGLIN, J.I. \& PROBST, J.L. Transports fluviaux de matières dissoutes et particulaires sur um basin versant em région tropicale: Le basin versant Du Niger au cours de La période 1990-1993. Science Géologique Bulletin. Strasbourg, v.49, n.1-4, p.25-45, 1996.

3. BORTOLETTO JUNIOR, M.J. Características hidrogeoquímicas e processos erosivos mecânicos e químicos nas bacias de drenagem dos rios Tietê e Piracicaba. 2004. 202 f. Tese (Doutorado) - Centro de Energia Nuclear na Agricultura, Universidade de São Paulo, Piracicaba, 2004.

4. CARPENTER, S.R.; CARACO, N.F.; CORREL, D.L.; HOWARTH, R.W.; SHARPLEY, A.N., SMITH, V.H. Non-point pollution of surface waters with phosphorus and nitrogen. Ecological Applications, Washington, v.8, p.559-568, 1998.

5. CONCEIÇÃO, F.T. \& BONOTTO, D.M. Relações hidroquímicas aplicadas à avaliação da qualidade de água e diagnóstico ambiental na bacia do Rio Corumbataí (SP). Geochimica Brasiliensis, v.16, n.1, p.1-21, 2002.

6. CONCEIÇÃO, F.T. \& BONOTTO, D.M. Use of $\mathrm{U}$-isotopes disequilibrium to evaluate the weathering rate and fertilizer-derived uranium at São Paulo State. Environmental Geology, v.44, n.4, p.408-418, 2003.

7. CONCEIÇÃO, F.T. \& BONOTTO, D.M. Weathering rates and anthropogenic influences in a sedimentary basin, São Paulo State, Brazil. Applied Geochemistry, v.19, n.4, p.575- 591, 2004.

8. CONCEIÇÃO, F.T.; SARDINHA, D.S.; SOUZA, A.D.G.; NAVARRO, G.R.B. Anthropogenic influences on annual flux of cations and anions at Meio Stream basin, São Paulo State, Brazil. Water Air and Soil Pollution, v. 205, n. 1-4, p. 79-91, 2010.

9. DIONEX CORPORATION. ICS-90 ion chromatography system operator's manual. California: Dionex Corporation, 2004. (Document n.031851, revision 4).

10. DREVER, J.I. The geochemistry of natural waters: surface and groundwater environments. 3. ed. Upper Saddle River, New Jersey: Prentice-Hall, 1997, 436 p.

11. EDET, A.; UKPONG, A.; NGANJE, T. Hydrochemical studies of Cross River Basin (southeastern
Nigeria) river system using cross plots, statistics and water quality index. Environmental Earth Sciences, v. 70, p. 3043-3056, 2013.

12. EDMOND, J.M. High precision determination of titration alkalinity and total carbon dioxide content of seawater by potentiometric titration. Deep-Sea Research. Part I: Oceanographic Research Papers, v.17, n.4, p.737-750, 1970.

13. GARRELS, R.M. \& MACKENZIE, F.T. Origin of the chemical composition of some springs and lakes. In: Equilibrium concepts in natural waters systems. Washington: American Chemical Society, Advances in Chemistry Series, 1967.

14. GIBBS, R.J. Mechanisms controlling world river water chemistry. Science, v.170, p.1088-1090, 1970.

15. GIBBS, R.J. The geochemistry of the Amazon River System. Part 1. The factors that control the salinity and composition and concentration of suspended solids. Geological Society of America Bulletin, v.78, p.12031232, 1967.

16. HALL, F.R. Dissolved solids-discharge relationships. 1. Mixing models. Water Resources Research, v.6, n.3, p. 845-850, 1970.

17. HALL, F.R. Dissolved solids-discharge relationships. 2. Applications to field data. Water Resources Research, v.7, n.3, p. 591-601, 1971.

18. HERMES L.C. \& SILVA, A.S. Avaliação da qualidade das águas: manual prático. Brasília: Embrapa Informação Tecnológica, 2004, 55p.

19. IBGE - INSTITUTO BRASILEIRO DE GEOGRAFIA E ESTATÍSTICA. Dados do Censo 2010. Diário Oficial da União, Brasília, DF, 04 nov. 2010. Disponível em: http://www.ibge.gov.br/censo2010/dados_divulgados/ind ex.php?uf=35. Acessado em: 03dez2010.

20. IPT - INSTITUTO DE PESQUISAS TECNOLÓGICAS. Relatório Zero da Bacia do Sorocaba e Médio Tietê - Atualização 2005 (Relatório Técnico $n^{\circ}$ 80). São Paulo: IPT, 2006.

21. JENKINS, A.; PETERS, N.E.; RHODE, A. Hydrology. In: MOLDAN, B. \& CERNY, J. Biogeochemistry of small catchments: a tool for environmental research. Chichester: John Wiley \& Sons, 1994.

22. KATTAN, Z. \& PROBST, J.L. Transport en suspension et en solution par la Moselle en période de 
crue. Actes des Journées d'Hydrologie, "Crues et inondations", Strasbourg, 16-18 october, p.143-167, 1986.

23. KÖPPEN, W.P. Climatologia: con un estudio de los climas de la tierra. 1. ed. em Español. México: Fondo de Cultura Economica, 478 p., 1948.

24. LARAQUE, A.; MOQUET, J.S.; ALKATTAN, R.; STEIGER, J.; MORA, A.; ADÈLE, G.; CASTELlANOS, B.; LAGANE, C. LOPEZ, J.L.; PEREZ, J.; RODRIGUEZ, M,; ROSALES, J. Seasonal variability of total disolved fluxes and origin of major disolved elements within a large tropical river: The Orinoco, Venezuela. Journal of South American Earth Sciences, v. 44, p. 4-17, 2013.

25. LI, S. \& ZHANG, Q. Geochemistry of the Upper Han River basin, China, 1: Spatial distribution of major ion compositions and their controlling factors. Applied Geochemistry, v. 23, p. 3535-3544, 2008.

26. MARTIN, J.M. \& MEYBECK, M. Elemental mass-balance of material carried by major world rivers. Marine Chemistry, Amsterdam, v.7, p.173-206, 1979.

27. MARTINI, I.P. \& CHESWORTH, W. Weathering, Soils and Paleosols. Elsevier Science Publications, Amsterdam, 1992.

28. MEYBECK, M. Global geochemical weathering of surficial rocks estimated from river disolved loads. American Journal of Science, v.287, p.401-428. 1987.

29. MEYBECK, M. Composition chimique des ruisseaux non pollués de France. Science Géologique Bulletin, Strasbourg v.39, p.3-77, 1986.

30. MORTATTI, J. Erosão na Amazônia: processos, modelos e balanço. Piracicaba, 1995. 155 f. Tese (LivreDocência) - Escola Superior de Agricultura "Luiz de Queiroz", Universidade de São Paulo.

31. MORTATTI, J.; PROBST, J.L.; FERNANDES, A.M.; MORTATTI, B.C.; OLIVEIRA, H. Influence of discharge on silicate weathering dynamics of the Tietê river basin: major cations and disolved silica approach. Geochimica Brasiliensis, v.22, n.1, p.15-26, 2008.

32. MORTATTI, J.; PROBST, J.L.; TARDY, Y. Avaliação do escoamento superficial na Bacia Amazônica através do transporte de material fluvial. Geochimica Brasiliensis, v.8, n.2, p.235-243, 1994.

33. MORTATTI, J.; VENDRAMINI, D.; OLIVEIRA, H. Avaliação da poluição doméstica fluvial na zona urbana do município de Piracicaba, SP, Brasil. Revista Ambiente e Água - An interdisciplinar Journal of Applied Science, v. 7, n. 2, p. 110-119, 2012.

34. OLIVEIRA, H.; MORTATTI, J.; MORAES, G.M.; VENDRAMINI, D.; CAMPOS, K.B.G. Caracterização hidrogeoquímica da carga dissolvida nas bacias dos rio Jundiaí e Capivari, São Paulo. Geociências, São Paulo, v. 33, n. 2, p. 278-297, 2014.
35. PROBST, J.L. Géochimie et Hydrologie de l'Érosion Continentale. Mécanisms, Bilan Global Actuel et Fluctuations au Cours des 500 Derniers millions d'annés. Sci. Géol., 94, Strasbourg, 161 p. 1992. 36. PROBST, J.L.; AMIOTTE-SUCHET, P.; TARDY, Y. Global continental erosion and fluctuations of atmospheric $\mathrm{CO}_{2}$ consumed during the last 100 years. Water-Rock Interaction, p.483-486, 1992b.

37. PROBST, J.L.; NKOUNKOU, R.R; KREMPP, G.; BRICQUET, J.P.; THIÉBAUX, J.P.; OLIVRY, J.C. Dissolved major elements exported by the Congo and the Ubangui rivers during the period 1987-1989. Journal of Hydrology, v.135, p.237-157, 1992a.

38. RIBANI, M.; BOTTOLI, C.B.G.; COLLINS, C.H.; JARDIM, I.C.S.F.; MELO, L.F.C. Validação em métodos cromatográficos e eletroforéticos. Química Nova, v.27, n.5, p.771-780, 2004.

39. ROSS, J.L.S. \& MOROZ, I.C. Mapa geomorfológico do Estado de São Paulo. São Paulo: Laboratório de Geomorfologia, Depto Geografia, FFLCHUSP, Laboratório de Cartografia Geotécnica - Geologia Aplicada - IPT, FAPESP. Mapas e Relatórios - SP, 1997. 40. ROSS, J.L.S. Geografia do Brasil. v. 3. São Paulo: EDUSP, 546 p., 1996.

41. SARDINHA, D.S.; CONCEIÇÃO, F.T.; BONOTTO, D.M.; SALLES, M.H.D.; ANGELUCCI, V.A. Avaliação de balanço anual de cátions e ânions na bacia do Alto Sorocaba (SP). Revista Brasileira de Geociências, v.38, n.4, p.730-740, 2008.

42. SILVA, G.M.P. Diagnóstico ambiental da bacia do rio Corumbataí: um estudo analítico. Rio Claro: Centro de Estudos Ambientais, Universidade Estadual Paulista, 1994. (Relatório)

43. STALLARD, R.F. \& EDMOND, J.M. Geochemistry of the Amazon Basin. 2. The influence of the geology and weathering environment on the dissolved load. Journal of Geophysical Research, Washington, v.88, p.9671-9688, 1983.

44. STALLARD, R.F. \& EDMOND, J.M. Geochemistry of the Amazon Basin. 3. Weathering chemistry and limits to dissolved inputs. Journal of Geophysical Research. Serie C, Washington, v.92, n. 8 p.8293-8302, 1987.

45. TARDY, Y.; BUSTILLO, V.; ROQUIM, C.; MORTATTI, J.; VICTORIA, R. The Amazon. Biogeochemistry applied to river basin management. Part I. Hydro-climatology, hydrograph separation, mass transfer balances, stable isotopes, and modelling. Applied Geochemistry, n.20, p.1746-1829, 2005.

Manuscrito recebido em: 12 de Novembro de 2014 Revisado e Aceito em: 05 de Setembro de 2016 